\title{
On the approximation of particular solution of nonhomogeneous linear differential equation with Legendre series
}

\author{
Nichaphat Patanarapeelert ${ }^{1 *}$ and Klot Patanarapeelert ${ }^{2}$ \\ ${ }^{1}$ Department of Mathematics, Faculty of Applied Science, King Mongkut's University of Technology North Bangkok, Bang \\ Sue district, Bangkok 10800 Thailand \\ ${ }^{2}$ Department of Mathematics, Faculty of Science, Silpakorn University, Mueang district, Nakhon Pathom 73000 Thailand \\ *E-mail: nichaphat.p@sci.kmutnb.ac.th
}

\begin{abstract}
In this study, we propose an approximation method for particular solutions of the nonhomogeneous second-order differential equations by truncated Legendre series. Particulary, the govern problem is a linear differential equation with constant coefficients. The choice of series solutions depends upon the complementary solutions and the approximate nonhomogeneous terms. An upper bound for the approximation error is formulated. Some examples are presented to demonstrate the validity of the proposed method.
\end{abstract}

Keywords: nonhomogeneous differential equations, particular solutions, Legendre polynomials, series, error bound

\section{Introduction}

Many problems in natural science such as physics, engineering and mathematical modeling are governed by differential equations (J ung et al., 2014). Solving such equations will lead to understanding the behaviors of the systems. Although solutions are known to be exist, there is an only few problems that can be solved for analytic solution. Several attempts are devoted to numerical method or approximation techniques to obtain the high accuracy of approximations ( $\mathrm{ung}$ et al., 2014).

Taylor series and orthogonal functions such as Chebyshev and Legendre polynomials are powerful tools for functions approximations in terms of polynomials (Gulsu et al., 2006; Wang and Xiang, 2012; Patanarapeelert and Varnasavang, 2013). As a by-product they can be used for approximating the solution of ordinary differential equations. Sezer and Gulsu (2010) proposed a numerical method based on the hybrid Legendre and Taylor polynomials for solving the high-order linear differential equations. Olagunju and Olaninejum (2012) formulated a trial solution for nonhomogeneous differential equations where Legendre polynomials are used as basis functions. Recently, J ung et al. (2014) proposed the method to solutions of second-order differential equations by using Tau method based on Legendre operational matrix.

In this paper, we present a method for approximating the particular solutions of nonhomogenous linear second-order differential equations. Rather than approximating as a whole we focus on in part, approximating particular solution by which the complementary solution is prior known. In doing so, we transfer the original problem into an approximate one 
by approximating the nonhomogeneous term with a truncated Legendre series expansion and hence assume a particular solution of the approximated problem in terms of finite Legendre series. Since the nonhomogeneous term and the particular solution are approximated as polynomials, we consider the cases in which they can be dependent on the corresponding complementary solution. We hypothesize that the accuracy of approximation depends on the highest degree of polynomial used in the series; the more number of terms are used, the more decreasing in error magnitude. To verify this we investigate the upper bound for the error between the exact solution and the estimation.

\section{Method of Finding Approximate Solution Based on Legendre Series}

Consider a nonhomogeneous linear second-order differential equation

$$
L[y]=a y^{\prime \prime}(t)+b y^{\prime}(t)+c y(t)=f(t)
$$

with the initial conditions

$$
y(0)=\alpha_{0}, y^{\prime}(0)=\beta_{0}
$$

where $a \neq 0, b, c$ are constants and $f(t)$ is a continuously differentiable function up to order $n$. The general solution for ( 1 ) is given by $y=y_{c}+y_{p}$ where $y_{c}$ is the complementary solution obtained from the associated homogeneous differential equation while $y_{p}$ is a particular solution. In order to find the approximation of $y_{p}$, we first approximate the nonhomogeneous term $f(t)$ as the truncated Legendre series as

$$
f(t)=\sum_{m=0}^{n} a_{m} P_{m}
$$

where $P_{0}=1$,

$$
\begin{aligned}
& P_{1}=t, \\
& P_{m+1}=\frac{1}{m+1}\left[(2 m+1) t P_{m}-m P_{m-1}\right] ; m=2, \ldots, n
\end{aligned}
$$

are the Legendre polynomials and the coefficients are denoted by

$$
a_{m}=\frac{2 m+1}{2} \int_{-1}^{1} f(t) P_{m}(t) d t
$$

where $-1 \leq t \leq 1$. Noting for generality that even $f(x)$ is defined on $\tau_{1} \leq x \leq \tau_{2}$, we can transform into $-1 \leq t \leq 1$ by using the relation $x=\frac{1}{2}\left[\left(\tau_{1}-\tau_{0}\right) t+\tau_{1}+\tau_{0}\right]$ (Ascher, 2008). We then obtain an approximated problem for equation (1) as 


$$
L[\hat{y}]=a \hat{y}^{\prime \prime}(t)+b \hat{y}^{\prime}(t)+c \hat{y}(t)=\sum_{m=0}^{n} a_{m} P_{m}
$$

with the initial conditions

$$
\hat{y}(0)=\gamma_{0}, \hat{y}^{\prime}(0)=\eta_{0}
$$

For this problem we suppose its solution in the form $\hat{y}=\hat{y}_{c}+\hat{y}_{p}$. It should be noted that the form of complementary solutions of equations (1) and (4) are similar. In order to solve for particular solution we assume that

$$
\hat{y}_{p}=\sum_{i=0}^{n+l} b_{i} P_{i}-\left(A P_{0}+B P_{1}\right)
$$

where the unknown parameters $l, A$ and $B$ depend on the form of complementary solution. The reason behind this is that the linearly independence of two solutions must be preserved (Rice and Do, 2012). To illustrate this clearly, we classify $l, A$ and $B$ as follows.

Case I. If there is no any term of polynomials appearing in homogeneous solution, then

$$
l=A=B=0
$$

Case II . If there is a polynomial consisting of only constant term appearing in homogeneous solution, then

$$
l=1, B=0, A=\sum_{m=0}^{\left[\frac{n}{2}\right]} \frac{(-1)^{m}(2 m) !}{2^{2 m}(m !)^{2}} b_{2 m}
$$

where $\left[\frac{n}{2}\right]=\frac{n}{2}$ for $n=0,2,4, \ldots$ and $\left[\frac{n}{2}\right]=\frac{n+1}{2}$ for $n=1,3,5, \ldots$.

Case III . If there is a polynomial of degree one appearing in homogeneous solution, then 


$$
l=2, A=\sum_{m=0}^{\left[\frac{n+2}{2}\right]} \frac{(-1)^{m}(2 m) !}{2^{2 m}(m !)^{2}} b_{2 m}, B=\sum_{m=0}^{\left[\frac{n}{2}\right]} \frac{(-1)^{m}(2 m+2) !}{2^{2 m+1} m !(m+1) !} b_{2 m+1}
$$

where $\left[\frac{n+2}{2}\right]=\frac{n+2}{2}$ for $n=0,2,4, \ldots$ and $\left[\frac{n+2}{2}\right]=\frac{n+1}{2}$ for $n=1,3,5, \ldots$.

In order to determine the coefficients $b_{i}$, we substitute (6) into (4), so that

$$
a \sum_{i=0}^{n+l} b_{i} P_{i}^{\prime \prime}+b\left(\sum_{i=0}^{n+l} b_{i} P_{i}^{\prime}-B P_{1}^{\prime}\right)+c\left(\sum_{i=0}^{n+l} b_{i} P_{i}-A P_{0}-B P_{1}\right)=\sum_{m=0}^{n} a_{m} P_{m} .
$$

The derivatives of Legendre polynomials can be written in the form of Legendre polynomials as

$$
\begin{gathered}
P_{n}^{\prime}=\sum_{m=0}^{n}(2 n-4 m-1) P_{n-2 m-1} ; n \geq 0\left(P_{n}=0 ; n \leq 0\right), \\
P_{n}^{\prime \prime}=\sum_{m=0}^{n}(2 n-4 m-1) \sum_{k=0}^{n-2 m-1}(2 n-4 m-4 k-3) P_{n-2 m-2 k-2} .
\end{gathered}
$$

Employing (11) and (12), we observe that equation (10) becomes the algebraic equation of Legendre polynomials. Finally, the values of $b_{i}$ are accomplished by equating the corresponding coefficients of $P_{i}$ and solving the system of algebraic equations.

\section{Upper Bound for Approximation Error}

In this section, we investigate an upper bound for the error between the exact solution and the approximated solution obtained from the method mentioned above.

Let $z=y-\hat{y}$ denotes the error of approximated solution, this quantity is then satisfied the differential equation

$$
L[z]=a z^{\prime \prime}(t)+b z^{\prime}(t)+c z(t)=E(t)
$$

where 


$$
E(t)=f(t)-\sum_{m=0}^{n} a_{m} P_{m}=\sum_{m=n+1}^{\infty} a_{m} P_{m} .
$$

We note that $E(t)$ is an error for approximating nonhomogeneous term. In order to determine the bounds for solution of (13), we follow the theory developed in previous work (Brauer, 1963). To be proceed, we first rewrite (13) in the form of system of first-order equations by letting $u(t)=z$ and $v(t)=d z / d t$. The resulting system is given by

$$
\begin{aligned}
& \frac{d u}{d t}=v \\
& \frac{d v}{d t}=\frac{E(t)-c u-b v}{a} .
\end{aligned}
$$

The above equations can be written in matrix notation by denoting $\mathbf{X}(t)=\left(\begin{array}{ll}u & v\end{array}\right)^{T}$ as a vector function. Equation (16) then becomes

$$
\frac{d \mathbf{X}}{d t}=\mathbf{f}(t, \mathbf{X})=A \mathbf{X}+\mathbf{e}
$$

where

$$
A=\left(\begin{array}{rr}
0 & 1 \\
-\frac{c}{a} & -\frac{b}{a}
\end{array}\right), \mathbf{e}=\left(\begin{array}{c}
0 \\
\frac{E(t)}{a}
\end{array}\right) .
$$

Next, we will determine a scalar function that is bounded above the right hand side of (17). Since

$$
\|\mathbf{f}(t, \mathbf{X})\| \leq\|A\|\|\mathbf{X}\|+\|\mathbf{e}\|
$$

where $\|$.$\| is a vector norm, and$ 


$$
\|\mathbf{e}\|=\left|\frac{E(t)}{a}\right| \leq \frac{1}{|a|} \sum_{m=n+1}^{\infty}\left|a_{m} P_{m}(t)\right| \leq \frac{1}{|a|} \sum_{m=n+1}^{\infty}\left|a_{m}\right|
$$

because $\left|P_{m}(t)\right| \leq 1[10]$, it follows that

$$
\|\mathbf{f}(t, \mathbf{X})\| \leq\|A\|\|\mathbf{X}\|+\frac{1}{|a|} \sum_{m=n+1}^{\infty}\left|a_{m}\right|
$$

Define a function

$$
\omega(r)=\|A\| r+\frac{1}{|a|} \sum_{m=n+1}^{\infty}\left|a_{m}\right|
$$

and noting that $r \equiv r(t)>0$ is a positive function. From (20), it is true that

$$
\|\mathbf{f}(t, \mathbf{X})\| \leq \omega(\|\mathbf{X}\|)
$$

Solving for $r(t)$ of an equation

$$
r^{\prime}=\omega(r)
$$

with $r(0)=\|\mathbf{X}(0)\|$ and $\omega(r)$ is given in (21), we have

$$
r(t)=\frac{e^{\|A\| t}}{|a|}\left(\frac{1-e^{-\|A\| t}}{\|A\|}\right) \sum_{m=n+1}^{\infty}\left|a_{m}\right|+e^{\|A\| t} r(0) .
$$

From the theory of upper bound for solutions of ordinary differential equations (Brauer, 1962), we can conclude that

$$
\|\mathbf{X}(t)\| \leq r(t)
$$


for all $t$. By means of vector norm, we arrive at the main result

$$
|y-\hat{y}| \leq r(t)
$$

The expression of $r(t)$ can be determined explicitly following (24). The difficulty arises however when attempting to calculate the summation $\sum\left|a_{m}\right|$. To facilitate the calculation some properties of truncated Legendre series expansion is introduced (Wang and Xiang, 2011). Since $f, f^{\prime}, f^{\prime \prime}, \ldots, f^{(n-2)}$ are continuous on $[-1,1]$ and if $\left\|f^{(n-1)}\right\|_{T}=V_{n-1}<\infty$ where

$$
\|\xi\|_{T}=\int_{-1}^{1} \frac{\left|\xi^{\prime}(t)\right|}{\sqrt{1-t^{2}}} d t
$$

for some $n \geq 2$, we apply the error bounds of truncated Legendre series expansion derived by Wang and Xiang (2011) to get that

$$
\sum_{m=n+1}^{\infty}\left|a_{m}\right| \leq \sum_{m=n+1}^{\infty} \frac{V_{n-1}}{\left(m-\frac{1}{2}\right)\left(m-\frac{3}{2}\right) \ldots\left(m-\frac{2 n-3}{2}\right)} \sqrt{\frac{\pi}{2(m-n)}}
$$

Thus,

$$
|y(t)-\hat{y}(t)| \leq E R B(t)
$$

where

$$
\operatorname{ERB}(t)=\frac{e^{\|A\| t}}{|a|}\left(\frac{1-e^{-\|A\| t}}{\|A\|}\right) \sum_{m=n+1}^{\infty}\left[\frac{V_{n-1}}{\left(m-\frac{1}{2}\right)\left(m-\frac{3}{2}\right) \ldots\left(m-\frac{2 n-3}{2}\right)} \sqrt{\frac{\pi}{2(m-n)}}\right]+e^{\|A\| t} r(0)
$$

with $n \geq 2$, is the upper bound for approximation error as desired. 


\section{Examples} section.

Here, we provide some examples to illustrate our method presented in the previous

Example 1 In this example, we find the general solution of the nonhomogeneous linear second order differential equation

$$
y^{\prime \prime}-y^{\prime}=\sin ^{-1} t
$$

The complementary solution of (31) is $c_{1}+c_{2} e^{t}$. Employing the proposed method, we rewrite the nonhomogeneous term in the form of Legendre series expansions as

$$
\sin ^{-1} t=a_{0} P_{0}+a_{1} P_{1}+a_{2} P_{2}+a_{3} P_{3}
$$

where $a_{0}=0, a_{1}=\frac{3 \pi}{8}, a_{2}=0, a_{3}=\frac{\pi}{8}$ (Patanarapeelert and Varnasavang, 2013).

From (32), the problem (31) is rewritten as

$$
\hat{y}^{\prime \prime}-\hat{y}^{\prime}=a_{0} P_{0}+a_{1} P_{1}+a_{2} P_{2}+a_{3} P_{3}
$$

From case II, we have $l=1, B=0$ since the complementary solution has a constant term. Therefore,

$$
\hat{y}_{p}=\sum_{i=0}^{4} b_{i} P_{i}-A P_{0}
$$

where

$$
A=\sum_{m=0}^{2} \frac{(-1)^{m}(2 m) !}{2^{2 m}(m !)^{2}} b_{2 m}
$$

After substituting (34) and (35) in (33), we have $b_{1}=-\frac{9 \pi}{4}, b_{2}=-\frac{41 \pi}{56}, b_{3}=-\frac{\pi}{8}, b_{4}=-\frac{\pi}{56}$. The expression of the particular solution (34) after rearranging terms in increasing powers of $t$ is

$$
\hat{y}_{p}=-\frac{7 \pi}{4} t-\frac{33 \pi}{32} t^{2}-\frac{5 \pi}{6} t^{3}-\frac{5 \pi}{64} t^{4} .
$$

Therefore, we finally obtain the approximate solution as 


$$
\hat{y}=c_{1}+c_{2} e^{t}-\frac{7 \pi}{4} t-\frac{33 \pi}{32} t^{2}-\frac{5 \pi}{6} t^{3}-\frac{5 \pi}{64} t^{4}
$$

We note that the method of undetermined coefficients is not applicable to linear equation (31) with such nonhomgeneous term. In addition, we could not find its particular solution by employing the method of variation of parameters analytically as well. Therefore, the above procedure can be used as alternative way to approximate the solution.

We further illustrate the results of an upper bound for the error between the exact solution and the approximated solution by the given following examples.

Example 2 Consider the nonhomogeneous linear second order differential equation

$$
y^{\prime \prime}(t)=e^{t}
$$

with the initial conditions

$$
y(0)=2, y^{\prime}(0)=1
$$

We start with the approximation

$$
e^{t}=a_{0} P_{0}+a_{1} P_{1}+a_{2} P_{2}
$$

where the coefficients of Legendre polynomials $a_{0}=1.1752, a_{1}=1.1036, a_{2}=0.3578$ are obtained from the orthogonal relation $a_{m}=\frac{2 m+1}{2} \int_{-1}^{1} e^{t} P_{m}(t) d t$. Hence, we now solve the problem

$$
\hat{y}^{\prime \prime}(t)=a_{0} P_{0}+a_{1} P_{1}+a_{2} P_{2}
$$

with the initial conditions

$$
\hat{y}(0)=2, \hat{y}^{\prime}(0)=1 \text {. }
$$

Equations (41) has a complementary solution

$$
\hat{y}_{c}=c_{1}+c_{2} t
$$


where $c_{1}, c_{2}$ are arbitrary constants.

Since there is the polynomials with degree one appeared in (43), we assume the particular solution for (41) as

$$
\hat{y}_{p}=\sum_{i=0}^{4} b_{i} P_{i}-\left(A P_{0}+B P_{1}\right)
$$

where

$$
\begin{aligned}
& A=\sum_{m=0}^{2} \frac{(-1)^{m}(2 m) !}{2^{2 m}(m !)^{2}} b_{2 m}=b_{0}-\frac{1}{2} b_{2}+\frac{3}{8} b_{4} \\
& B=\sum_{m=0}^{1} \frac{(-1)^{m}(2 m+2) !}{2^{2 m+1} m !(m+1) !} b_{2 m+1}=b_{1}-\frac{3}{2} b_{3} .
\end{aligned}
$$

Substituting (44) in (41) and employing (12), we get

$$
\left(3 b_{2}+10 b_{4}\right) P_{0}+15 b_{3} P_{1}+35 b_{4} P_{2}=a_{0} P_{0}+a_{1} P_{1}+a_{2} P_{2}
$$

Comparing the coefficients of $P_{i} ; i=0,1,2$, we obtain

$$
b_{2}=\frac{1}{3} a_{0}-\frac{2}{21} a_{2}, b_{3}=\frac{1}{15} a_{1}, b_{4}=\frac{1}{35} a_{2} \text {. }
$$

After rearranging, we find the particular solution (44) in the form

$$
\hat{y}_{p}=\left(\frac{a_{0}}{2}-\frac{a_{2}}{4}\right) t^{2}+\frac{a_{1}}{6} t^{3}+\frac{a_{2}}{8} t^{4} .
$$

Therefore, the general solution of equation (41) is

$$
\hat{y}=c_{1}+c_{2} t+\left(\frac{a_{0}}{2}-\frac{a_{2}}{4}\right) t^{2}+\frac{a_{1}}{6} t^{3}+\frac{a_{2}}{8} t^{4} .
$$


Under the conditions (42), the approximate solution for the original equation (38) is

$$
\hat{y}=2+t+0.4981 t^{2}+0.1839 t^{3}+0.0447 t^{4} \text {. }
$$

While, the exact solution of (41) is

$$
y=1+t+e^{t}
$$

Next, we find the upper bound for the absolute error between (50) and (51) by using (30). For the problem (38), $\quad a=1, b=c=0$. Therefore, $A=\left(\begin{array}{ll}0 & 1 \\ 0 & 0\end{array}\right)$ and $\|A\|=1$. Calculating numerically under the tolerance of order $10^{-5}$, we find that

$$
\sum_{m=3}^{\infty} \frac{V_{1}}{\left(m-\frac{1}{2}\right)} \sqrt{\frac{\pi}{2(m-2)}}=8.2260
$$

where $V_{1}=\int_{-1}^{1} \frac{e^{t}}{\sqrt{1-t^{2}}} d t=3.9775$. Here, $r(0)=0$. Therefore, the upper bound is

$$
\operatorname{ERB}(t)=8.2260 e^{t}\left(1-e^{-t}\right)
$$

We then conclude that $|y(t)-\hat{y}(t)| \leq 8.2260 e^{t}\left(1-e^{-t}\right)$.

Table 1 shows the accuracy of approximations including with errors and error bounds. The plots of the exact solution, the approximate one, and $\hat{y}(t) \pm E R B(t)$ are shown in Figure 1 . In this example we eliminate the effect of initial condition by choosing the same values as the original problem. However, the magnitude of errors is quite large. This may be caused by the number of terms used in approximation is low. We observe that order in magnitude of errors does not agree with the order of magnitude of errors in function approximation (see Table 2). This result implies that the accuracy of approximate nonhomogeneous term does not necessarily guarantee the accuracy of approximate solutions. 


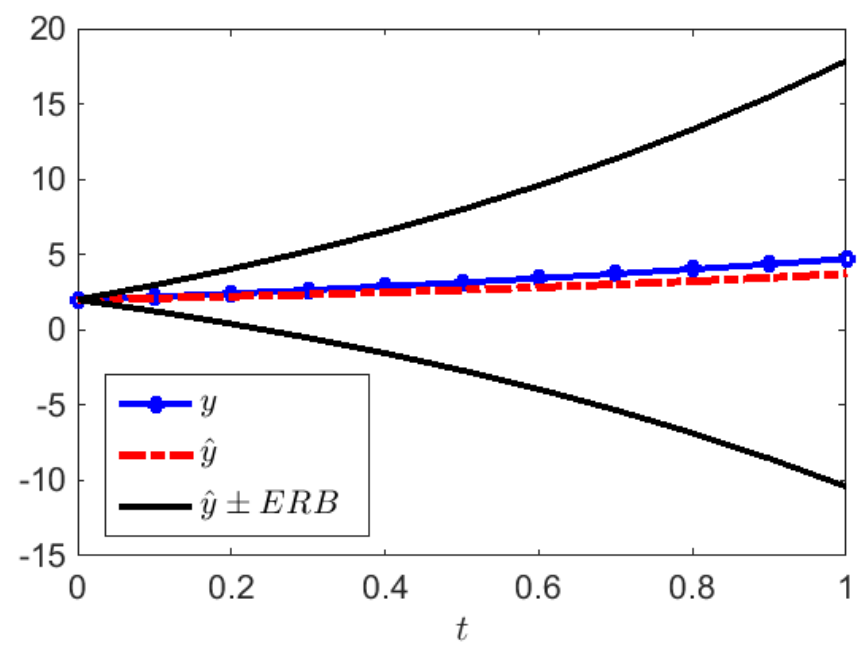

Figure 1. Plots of $y, \hat{y}, \hat{y}+E R B$, and $\hat{y}-E R B$ of Example 2 .

Table 1. The comparisons between the exact solution and the approximation for Example 2.

\begin{tabular}{ccccc}
\hline$t$ & $y$ & $\hat{y}$ & $|y-\hat{y}|$ & $E R B$ \\
\hline 0 & 2 & 2 & 0 & 0 \\
0.2 & 2.4214 & 2.2215 & 0.1999 & 1.8213 \\
0.4 & 2.8918 & 2.4926 & 0.3992 & 4.0457 \\
0.6 & 3.4221 & 2.8248 & 0.5973 & 6.7627 \\
0.8 & 4.0255 & 3.2312 & 0.7943 & 10.0813 \\
1 & 4.7183 & 3.7267 & 0.9916 & 14.1346 \\
\hline
\end{tabular}


Table 2. The errors between the nonhomogeneous term and the truncated Legendre series expansion for Example 2.

\begin{tabular}{cccc}
\hline$t$ & $e^{t}$ & $\sum_{m=0}^{2} a_{m} P_{m}$ & $\left|e^{t}-\sum_{m=0}^{2} a_{m} P_{m}\right|$ \\
\hline 0 & 1.0000 & 0.9963 & 0.0037 \\
0.2 & 1.2214 & 1.2385 & 0.0171 \\
0.4 & 1.4918 & 1.5236 & 0.0318 \\
0.6 & 1.8221 & 1.8517 & 0.0296 \\
0.8 & 2.2255 & 2.2227 & 0.0029 \\
1 & 2.7183 & 2.6366 & 0.0817 \\
\hline
\end{tabular}

Example 3 Consider the following differential equation

$$
y^{\prime \prime}-5 y^{\prime}+6 y=\sin t
$$

with the initial conditions

$$
y(0)=1, y^{\prime}(0)=\frac{1}{2}
$$

Here, we set

$$
\sin t=\sum_{m=0}^{5} a_{m} P_{m}
$$

where $a_{0}=a_{2}=a_{4}=0, a_{1}=0.9035, a_{3}=-0.0630, a_{5}=0.0010$. Therefore, we have the approximate problem as

$$
\hat{y}^{\prime \prime}-5 \hat{y}^{\prime}+6 \hat{y}=a_{0} P_{0}+a_{1} P_{1}+a_{2} P_{2}+a_{3} P_{3}+a_{4} P_{4}+a_{5} P_{5}
$$

with the initial condition 


$$
\hat{y}(0)=1, \hat{y}^{\prime}(0)=\frac{1}{2}
$$

A complementary solution for (55) is given by

$$
\hat{y}_{c}=c_{1} e^{2 t}+c_{2} e^{3 t}
$$

where $c_{1}, c_{2}$ are arbitrary constants.

Since there has no any polynomial term in (57), we assume the particular solution for (55) as

$$
\hat{y}_{p}=\sum_{i=0}^{5} b_{i} P_{i}
$$

To find $b_{i}$, we substitute (58) and use (11), (12) to (55) and then equate the coefficients of $P_{i} ; i=0,1, \ldots, 5$, to get a linear system of equations. After some calculations, we have

$$
\begin{aligned}
& b_{5}=\frac{a_{5}}{6}, b_{4}=\frac{a_{4}+45 b_{5}}{6}, b_{3}=\frac{a_{3}+35 b_{4}-63 b_{5}}{6}, \\
& b_{2}=\frac{a_{2}+25 b_{3}-35 b_{4}+25 b_{5}}{6}, b_{1}=\frac{a_{1}+15 b_{2}-15 b_{3}+15 b_{4}-42 b_{5}}{6}, \\
& b_{0}=\frac{a_{0}+5 b_{1}-3 b_{2}+5 b_{3}-10 b_{4}+5 b_{5}}{6} .
\end{aligned}
$$

The solution of initial value problem (55)-(56) is

$$
\begin{aligned}
\hat{y}= & c_{1} e^{2 t}+c_{2} e^{3 t}+b_{0}-\frac{1}{2} b_{2}+\frac{3}{8} b_{4}+\left(b_{1}-\frac{3}{2} b_{3}+\frac{15}{8} b_{5}\right) t \\
& +\left(\frac{3}{2} b_{2}-\frac{15}{4} b_{4}\right) t^{2}+\left(\frac{5}{2} b_{3}-\frac{35}{4} b_{5}\right) t^{3}+\frac{35}{8} b_{4} t^{4}+\frac{63}{8} b_{5} t^{5}
\end{aligned}
$$

where 


$$
\begin{aligned}
& c_{1}=-3 b_{0}+b_{1}+\frac{3}{2} b_{2}-\frac{3}{2} b_{3}-\frac{9}{8} b_{4}+\frac{15}{8} b_{5}+\frac{5}{2} \\
& c_{2}=2 b_{0}-b_{1}-b_{2}+\frac{3}{2} b_{3}+\frac{3}{4} b_{4}-\frac{15}{8} b_{5}-\frac{3}{2} .
\end{aligned}
$$

To see how accurate the approximation is, we find the exact solution of (52)-(53). It is given by

$$
y=\frac{23}{10} e^{2 t}-\frac{7}{5} e^{3 t}+\frac{1}{10} \sin t+\frac{1}{10} \cos t
$$

The upper bound for the absolute error for this example can be obtained in similar way. We first determine $A=\left(\begin{array}{rr}0 & 1 \\ -6 & 5\end{array}\right)$ and $\|A\|=\sqrt{61}$ and

$$
V_{4}=\int_{-1}^{1} \frac{\cos t}{\sqrt{1-t^{2}}} d t=2.4039
$$

By calculating numerically under the tolerance of order $10^{-5}$, we find that

$$
\sum_{m=6}^{\infty} \frac{V_{4}}{\left(m-\frac{1}{2}\right)\left(m-\frac{3}{2}\right)\left(m-\frac{5}{2}\right)\left(m-\frac{7}{2}\right)} \sqrt{\frac{\pi}{2(m-5)}}=0.0207
$$

We note that $r(0)=0$. Therefore, the upper bound is

$$
\operatorname{ERB}(t)=\frac{0.0207}{\sqrt{61}} e^{\sqrt{61 t}}\left(1-e^{-\sqrt{61} t}\right) .
$$

Thus, $|y(t)-\hat{y}(t)| \leq \frac{0.0207}{\sqrt{61}} e^{\sqrt{61 t}}\left(1-e^{-\sqrt{61} t}\right)$.

We present the accuracy of approximations including with errors and error bounds in Table 3. The plots of the exact and the approximate solutions, and $\hat{y}(t) \pm E R B(t)$ are shown in Figure 2. Here, we choose similar initial conditions for both problems. As opposed to previous example, the number of terms used in approximation is larger. This results in the small errors presented in approximate nonhomogeneous term (see Table 4). Also, the errors of approximate solution are low comparing with the previous example. 


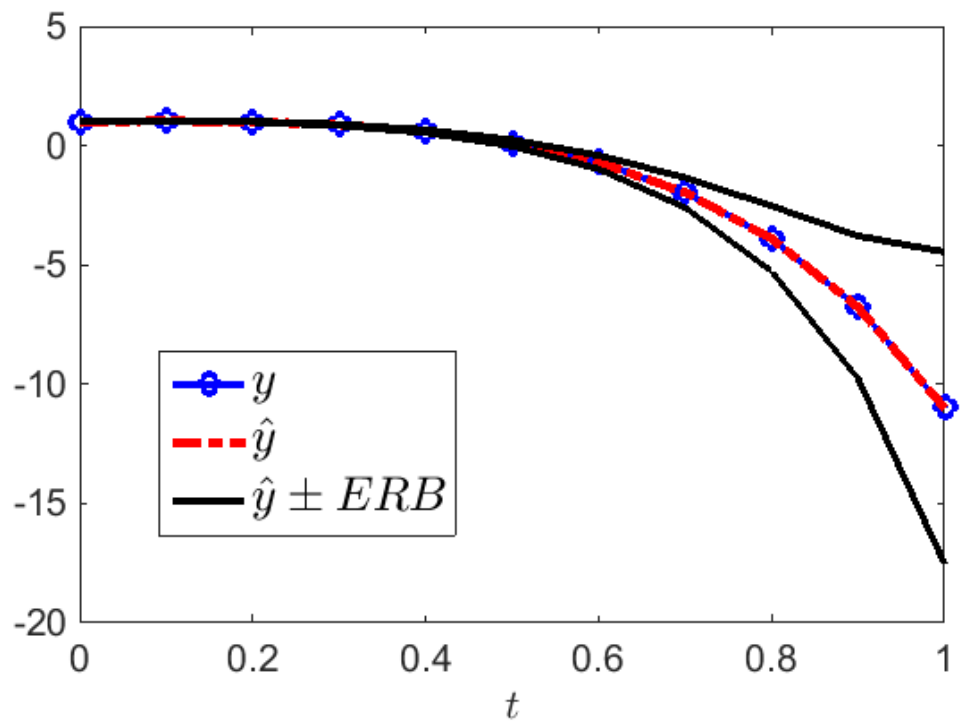

Figure 2. Plots of $y, \hat{y}, \hat{y}+E R B$, and $\hat{y}-E R B$ of Example 3 .

Table 3. The comparisons between the exact solution and the approximation for Example 3.

\begin{tabular}{ccccc}
\hline$t$ & $y$ & $\hat{y}$ & $|y-\hat{y}|$ & $E R B$ \\
\hline 0 & 1 & 1 & 0 & 0 \\
0.2 & 0.9981041 & 0.9981039 & 0.0000002 & 0.0099881 \\
0.4 & 0.6016284 & 0.6016264 & 0.0000020 & 0.0576174 \\
0.6 & -0.6942397 & -0.6942475 & 0.0000078 & 0.2847415 \\
0.8 & -3.8990661 & -3.8990875 & 0.0000214 & 1.3678025 \\
1 & -10.9867453 & -10.9867951 & 0.0000498 & 6.5324726
\end{tabular}


Table 4. The errors between the nonhomogeneous term and the truncated Legendre series expansion for Example 3.

\begin{tabular}{cccc}
\hline$t$ & $\sin t$ & $\sum_{m=0}^{5} a_{m} P_{m}$ & $\left|\sin t-\sum_{m=0}^{5} a_{m} P_{m}\right|$ \\
\hline 0 & 0 & 0 & 0 \\
0.2 & 0.1986693 & 0.1986475 & 0.0000218 \\
0.4 & 0.3894183 & 0.3893906 & 0.0000277 \\
0.6 & 0.5646425 & 0.5646274 & 0.0000151 \\
0.8 & 0.7173561 & 0.7173605 & 0.0000044 \\
1 & 0.8414710 & 0.8415000 & 0.0000290 \\
\hline
\end{tabular}

\section{Conclusion}

In summary, we proposed an approximation technique for solving the linear secondorder differential equation with nonhomogeneous term. The method presented here is different from previous works since it aims to approximate only the particular solution. It is believed that approximation in part may confine potentially the propagation of errors. The present method takes an advantage from approximating function with Legendre series by assuming series solution with the same degree as of approximate nonhomogeneous term. Since we are approximating the particular solution we improve the method by classifying the forms of series according to its complementary solution. This is possible in general when solving the equation with the traditional technique. To insure that the approximating results should not be divergent from the exact solution we derived the bound of approximation errors.

The expression of error bound shows the dependency of initial conditions and the degree of constructed series. We observe that however the derived error bound is exponentially increased with $t$ (the independent variable). This effect dominates all other dependent factors if $t$ is large. We argue that the reason behind this is that the method used for derivation is based upon the initial value problem (see Brauer (1963)). Thus, under the derived error bound, the approximation is locally (small interval of $t$ ) rather than globally. Importantly, we see that the error bound indicates how the precise approximation depends on the number of terms in constructed series. In the provided examples, the error bounds are exponentially increased with $t$. However, by increasing the number of terms of series we can expect the reduction in magnitude of errors (see Figure 3). It is observed that if $V_{n}$ in (27) is nonincreasing with $n$, the dependence on number of terms is only present by the series 


$$
\sum_{m=n+1}^{\infty} \frac{1}{\left(m-\frac{1}{2}\right)\left(m-\frac{3}{2}\right) \ldots\left(m-\frac{2 n-3}{2}\right)} \sqrt{\frac{\pi}{2(m-n)}}
$$

which is decreased with $n$.

The proposed method can be extended to the higher order of nonhomogeneous differential equations with constant coefficients and could be the first step of error analysis for the more complex problem. As mentioned, solving for analytic solution of differential equations is difficult even for linear nonhomogeneous problem. The method present here is as alternative, especially when the nonhomogeneous term is given in complex form. Nevertheless, there are some gaps that can be considered for the possible future work such as the derivation of error bounds for boundary value problems, the use of numerical interpolations for approximate function based on both Legendre and Chebyshev series. In addition, based on this method the possible extension should be the approximation for the differential equations with variable coefficients.

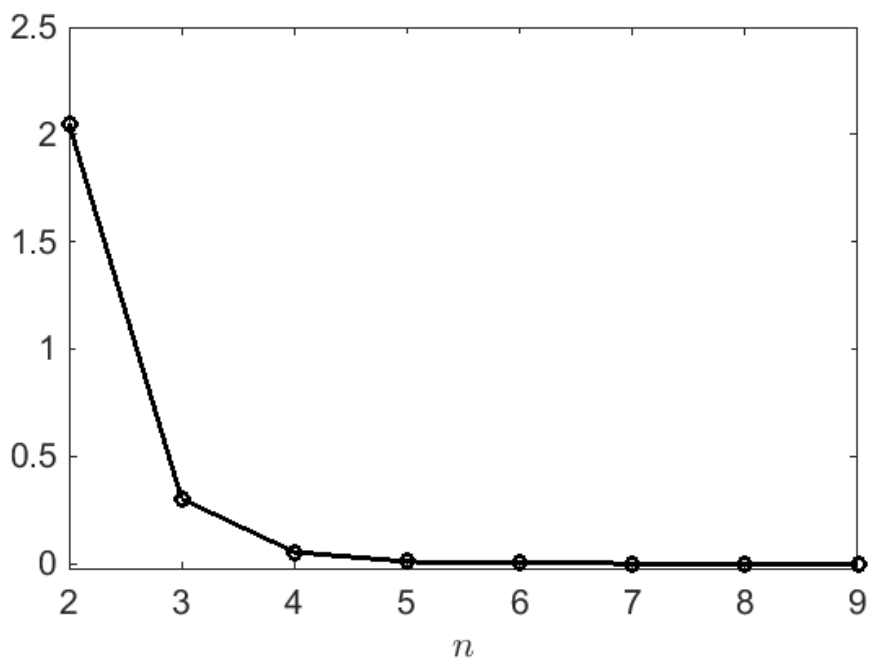

Figure 3. Plot of series (63) with respect to $n$.

\section{References}

Andrews L.C., (1992) Special Functions of Mathematics for Engineers, 2nd edition, McGraw-Hill, New York, U.S.A., 275 P.

Ascher U., (2008) "Methods and Concepts for ODEs", pp. 77-79, In: Ghattas O. (Ed), Numerical Methods for Evolutionary Differential Equations, Society for Industrial and Applied Mathematics, Philadelphia, U.S.A.

Brauer F., (1963) Bounds for Solutions of Ordinary Differential Equations. Proc. Amer. Math. Soc., 14: 36-43. 
Gulsu M., Sezer M. and Tanay B., (2006) A Matrix Method for Solving High-Order Linear Difference Equaitons with Mixed Argument Using Hybrid Legendre and Taylor Polynomials. J. Franklin Inst., 343: 647-659.

J ung C.Y., Liu Z, Rafiq A., Ali F. and Kang S.M., (2014) Solution of Second Order Linear and Nonlinear Ordinary Differential Equations using Legendre Operational Matrix of Differentiation. Int. J. Pure Appl. Math., 93(2): 285-295.

Olagunju A.S. and Olaniregun D.G., (2012) Legendre-Coefficients Comparison Methods for the Numerical Solution of a Class of Ordinary Differential Equations. IOSR-J M., 2(2): 14-19.

Patanarapeelert N. and Varnasavang V., (2013) Comparison Study of Series Approximation and Convergence between Chebyshev and Legendre Series. Appl. Math. Sci., 7(65): 32253237.

Rice G.R. and Do D.D., (2012) Applied Mathematics and Modeling for Chemical Engineers, 2nd edition, John Wiley \& Sons Inc., New J ersey, U.S.A., 400 P.

Sezer M. and Gulsu M., (2010) Solving High-Order Linear Differential Equations by a Legendre Matrix Method Based on Hybrid Legendre and Taylor Polynomials. NUMER. METH. PART. D. E., 26(3): 647-661.

Wang H. and Xiang S., (2011) On the Convergence Rates of Legendre Approximation. Math. Comput., 81(278): 861-877. 